\title{
EMPREGO DO TRATAMENTO VIA FLUIDO SUPERCRÍTICO NA ADSORÇÃO DE COBALTO UTILIZANDO QUITINA
}

\author{
J. M. CUNHA ${ }^{1}$, G. F. DÖRTZBACHER ${ }^{1}$, D. A. BERTUOL ${ }^{2}$, E. H. TANABE ${ }^{2}$ e G. L. \\ DOTTO $^{2}$
}

\begin{abstract}
${ }^{1}$ Universidade Federal de Santa Maria, Curso de Engenharia Química
${ }^{2}$ Universidade Federal de Santa Maria, Departamento de Engenharia Química

E-mail para contato: jeaninecunha@gmail.com; guilherme_dotto@yahoo.com.br

RESUMO - O cobalto é um metal tóxico presente em efluentes de indústrias metalúrgicas e eletrônicas. Entre as operações para sua recuperação está a adsorção. A quitina é um resíduo da indústria pesqueira, natural e abundante. Apresenta potencial como adsorvente de metais, com possibilidade de reuso. Estudou-se a influência da massa de adsorvente e tempo de contato. No estudo cinético, utilizaram-se os modelos de pseudo-primeira ordem e pseudo-segunda ordem, sendo o segundo o adequado para a representação do comportamento cinético. Para o equilíbrio, utilizaram-se os modelos de Freundlich e Langmuir, sendo o último o que melhor adaptou-se aos dados experimentais. A capacidade de adsorção estimada foi de $50 \mathrm{mg} \mathrm{g}^{-1}$ para a casca in natura e $101 \mathrm{mg} \mathrm{g}^{-1}$ para a modificada via $\mathrm{CO}_{2}$ supercrítico, indicando que o tratamento foi benéficona faixa de trabalho.
\end{abstract}

\section{INTRODUÇÃO}

Devido às crescentes aplicações, a presença de cobalto em efluentes industriais tornouse um objeto de preocupação. É um metal tóxico, utilizado em indústrias metalúrgicas e de eletrônicos. No meio ambiente, sofre bioacumulação e aumento da toxicidade ao longo da cadeia alimentar. (Javed et al., 2007). Entre os procedimentos para remoção de metais pesados de efluentes estão a troca iônica e a precipitação. Entretanto, esses métodos têm altos custos e requerem uso de químicos para recuperação dos metais (Franco et al., 2004). Uma das operações mais utilizadas para remoção de metais de efluentes é a adsorção em carvão ativado. É um material eficiente, porém com altos custos e dificuldade de regeneração do mesmo (Figueiredo et al., 2005). Estudos direcionam-se a adsorventes alternativos e de baixo custo. O potencial da quitina como material adsorvente de metais é ainda pouco estudado, mas mostra resultados promissores (Longhinotti et al., 1996).

A quitina é um biopolímero obtido industrialmente como subproduto de cascas de camarão e caranguejo. É o segundo biopolímero mais abundante no planeta. Pode ser isolada através de operações de desmineralização, desproteinização e desodorização. Sua atração está em sua biodegradabilidade, capacidade de regeneração, disponibilidade e baixo custo (Prashanth e Tharanathan, 2007). 
A quitina possui baixa área superficial, o que limita sua aplicação. O tratamento supercrítico é uma alternativa para melhorar as propriedades adsorventes da quitina. É um método rápido e limpo. A sua baixa viscosidade e ausência de tensão superficial contribuem para penetração do fluído na matriz sólida. A alta pressão pode causar o rompimento e expansão da estrutura da quitina, aumentando os sítios de adsorção, a área superficial e a porosidade do material (Santos, 2011).

O presente trabalho teve como objetivo investigar a adsorção de cobalto em quitina in natura e modificada via $\mathrm{CO}_{2}$ supercrítico.

\section{MATERIAL E MÉTODOS}

\subsection{Preparo e obtenção do adsorbato/adsorvente}

As soluções do adsorbato foram preparadas com sulfato de cobalto hepta-hidratado (99\%, VetecQuimica Fina LTDA) e água deionizada. A quitina foi obtida através de resíduos de camarão (Penaeus brasiliensis) e peneirada, utilizando-se partículas com diâmetro médio de $125 \mu \mathrm{m}$. Em seguida, 5.00g do material receberam tratamento via $\mathrm{CO}_{2}$ supercrítico a $35^{\circ} \mathrm{C}$ e 200 bar durante 1 hora (condições determinadas por testes preliminares) em um reator de inox, acoplado a um cilindro de $\mathrm{CO}_{2}$ e uma bomba de alta pressão (500D, Teledyne Isco, EUA).Os materiais foram caracterizados em relação à área superficial (Quantachrome Instruments, New Win2, EUA) e microscopia eletrônica de varredura (Jeol, JSM-6610LV, Japão).

\subsection{Ensaios de adsorção}

Os experimentos de adsorção de cobalto em quitina foram realizados em batelada, sob agitação e temperatura constantes $\left(120 \mathrm{rpm}, 30^{\circ} \mathrm{C}\right)$, com o auxílio de um banho com agitação do tipo Dubnoff (Marconi LTDA). O pH ótimo foi determinado por testes preliminares e fixado em 6. O volume de solução de cobalto foi fixado em $50 \mathrm{~mL}$, com concentração de 100 $\mathrm{mg} \mathrm{L}{ }^{-1}$. Variou-se o tempo de agitação $(0-300 \mathrm{~min})$ para o estudo cinético. No estudo do equilíbrio, colocou-se $0,05,0,1,0,2,0,3,0,4$ e $0,5 \mathrm{~g}$ de adsorvente, em cada frasco. Ao final, a quitina e o metal adsorvido foram separados da fase líquida por filtração. As amostras da fase líquida, contendo a concentração remanescente de cobalto, foram diluídas e analisadas por absorção atômica em chama (Agilent). As capacidades de adsorção de cobalto no tempo $t\left(q_{t}\right)$, no equilíbrio $\left(q_{e}\right)$, expressas em $\mathrm{mg} \mathrm{g}^{-1}$ foram obtidos pelas Eqs.(1), (2):

$$
\begin{aligned}
& q_{t}=\frac{\left(C_{0}-C_{t}\right)}{m} V \\
& q_{e}=\frac{\left(C_{0}-C_{e}\right)}{m} V
\end{aligned}
$$

Onde $C_{0}, C_{t}$, e $C_{e}\left(\mathrm{mg} \mathrm{L}^{-1}\right)$ são a concentrações de cobalto na fase líquida inicial, no tempo t, e no equilíbrio, respectivamente; $\mathrm{m}$ (g) é a massa de adsorvente; e V (L) é o volume de solução. 


\subsection{Cinética de adsorção}

Os dados cinéticos foram ajustados aos modelos de pseudo-primeira e pseudo-segunda ordem (Equações 3 e 4). Onde $q_{1}$ e $q_{2}\left(\mathrm{mgg}^{-1}\right)$ são as capacidades teóricas de adsorção; $k_{1}$ $\left(\mathrm{min}^{-1}\right)$ e $k_{2}\left(\mathrm{~g} \mathrm{mg}^{-1} \cdot \mathrm{min}^{-1}\right)$ são as constantes cinéticas de pseudo-primeira ordem e pseudosegunda ordem, respectivamente.

$$
\begin{array}{r}
q_{t}=q_{1}\left(1-\exp \left(-k_{1} t\right)\right) \\
q_{t}=\frac{t}{\left(1 / k_{2} q_{2}^{2}\right)+\left(t / q_{2}\right)}
\end{array}
$$

\subsection{Equilíbrio de adsorção}

Os dados experimentais obtidos no estudo do equilíbrio foram ajustados nos modelos de isotermas de Freundlich (Equação 5) e Langmuir (Equação 6). Sendo $k_{F}$ e $n_{F}$ as constantes de Freundlich; $k_{L}$ a constante de Langmuir $\left(\mathrm{L} \mathrm{mg}^{-1}\right)$ e $q_{m}$ a capacidade máxima de adsorção (mg $\left.\mathrm{g}^{-1}\right)$.

$$
\begin{aligned}
& q_{e}=k_{F} C_{e}^{1 / n_{F}} \\
& q_{e}=\frac{q_{m} k_{L} C_{e}}{1+\left(k_{L} C_{e}\right)}
\end{aligned}
$$

\section{RESULTADOS E DISCUSSÃO}

\subsection{Caracterização da quitina}

A Figura 1 mostra as imagens obtidas em MEV da quitina antes e depois de aplicado o tratamento supercrítico. Observa-se uma grande mudança na superfície do material, passando de uma estrutura regular antes do tratamento para uma totalmente irregular após o mesmo, com presença de rugosidades e protuberâncias. A área superficial da quitina aumentou de 4,0 $\mathrm{m}^{2} \mathrm{~g}^{-1}$ para $11,3 \mathrm{~m}^{2} \mathrm{~g}^{-1}$. As modificações na superfície podem ser atribuídas à alta pressão utilizada e as interações entre a quitina e o $\mathrm{CO}_{2}$.

Figura 1 - Imagens de MEV da quitina antes e após o tratamento.
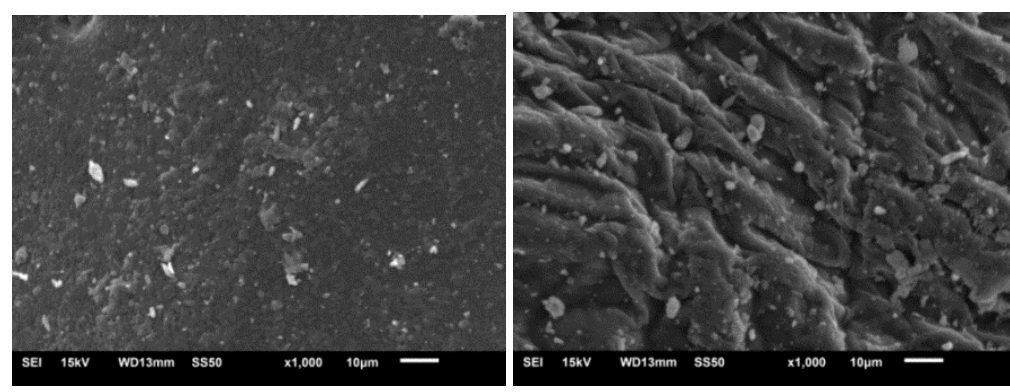


\subsection{Estudo cinético}

Os dados obtidos no estudo cinético estão representados na Figura 2. Em ambos os experimentos, o equilíbrio foi atingido por volta de 300 minutos. $\mathrm{O}$ material tratado chegou a uma capacidade de adsorção no equilíbrio de cerca $31 \mathrm{mg} \mathrm{g}^{-1}$ enquanto in natura atingiu aproximadamente $24 \mathrm{mg} \mathrm{g}^{-1}$. O tempo para a curva atingir a zona de equilíbrio é semelhante a outros adsorventes para íons metálicos (Spinelli et al., 2005).

Figura 2 - Curva cinética de adsorção (0,05 g de adsorvente).

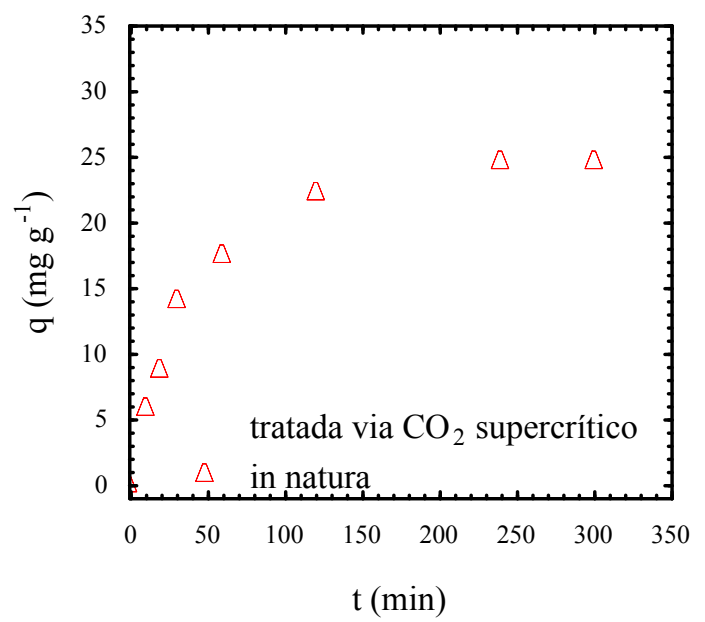

Os parâmetros obtidos no ajuste estão representados na Tabela 1.

Tabela 1 - Modelos cinéticos.

\begin{tabular}{ccc}
\hline \multicolumn{3}{c}{ Pseudo-primeira ordem } \\
\hline$q_{1}\left(\mathrm{mg} \mathrm{g}^{-1}\right)$ & in natura & Tratada \\
$k_{1}\left(\mathrm{~min}^{-1}\right)$ & 24,40 & 29,35 \\
$\mathrm{R}^{2}$ & 0,024 & 0,633 \\
$\mathrm{EMR}(\%)$ & 0,9838 & 0,9611 \\
& 6,09 & 7,75 \\
\hline$q_{2}\left(\mathrm{mg} \mathrm{g}^{-1}\right)$ & in natura & Tratada \\
$k_{2}\left(\mathrm{~g} \mathrm{mg}^{-1} \mathrm{~min}^{-1}\right)$ & 28,4 & 31,69 \\
$\mathrm{R}^{2}$ & 0,0063 & 0,0032 \\
$\mathrm{EMR}(\%)$ & 0,9877 & 0,9932 \\
& 4,97 & 3,22 \\
\hline
\end{tabular}

O modelo de pseudo-segunda ordem foi o mais adequado para representar a cinética de adsorção, apresentando $\mathrm{R}^{2}>0,98$ e EMR $<5 \%$, em ambos os casos. $\mathrm{O}$ valor de $q_{2}$ (capacidade de adsorção de acordo com o modelo de pseudo-segunda ordem) passou de 28,4 para 31,7 mg 
$\mathrm{g}^{-1}$ após a modificação. Esse modelo vem sendo utilizado para descrever a cinética de adsorção de íons metálicos (Spinelli et al.,2005);

\subsection{Estudo do equilíbrio}

A Figura 3 demonstra os dados da capacidade de adsorção para os dois materiais em função da massa de absorbato por massa de adsorvente utilizado. As curvas de equilíbrio apresentam comportamento semelhante, característico de adsorção em monocamada, indicando que a modificação no adsorvente não alterou o tipo de interação entre adsorbato/adsorvente. Entretanto, a quitina modificada apresentou uma maior capacidade de adsorção experimental, chegando a $44 \mathrm{mg} \mathrm{g}^{-1}$.

Figura 3 - Curvas do equilíbrio de adsorção.

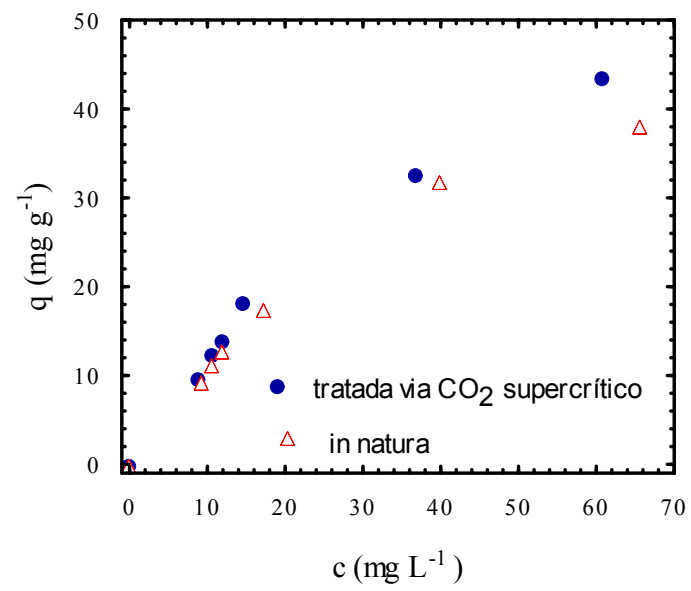

Tabela 2 - Modelos de equilíbrio.

\begin{tabular}{lcc}
\hline & Freundlich \\
\hline & in natura & Tratada \\
\cline { 2 - 3 }$k_{F}$ & 5,04 & 2,87 \\
$1 / n_{F}$ & 0,49 & 1,52 \\
$\mathrm{R}^{2}$ & 0,9873 & 0,9729 \\
EMR $(\%)$ & 6,62 & 11,97 \\
\hline \multicolumn{3}{c}{ Langmuir } \\
$q_{m}\left(\mathrm{mg} \mathrm{L}^{-1}\right)$ & in natura & Tratada \\
$\left.k_{L}(\mathrm{~L} \mathrm{mg})^{-1}\right)$ & 50,0 & 101,3 \\
$\mathrm{R}^{2}$ & 0,0459 & 0,1364 \\
EMR $(\%)$ & 0,9989 & 0,9939 \\
\hline
\end{tabular}

Os parâmetros obtidos nos ajustes das curvas de equilíbrio estão dispostos na Tabela 2.

Observa-se que o modelo de Langmuir foi o que melhor representou tal comportamento, com menores valores de EMR e valores de $\mathrm{R}^{2}$ mais próximos da unidade. $\mathrm{A}$ capacidade de adsorção estimada na faixa de trabalho estudada passou de $50,0 \mathrm{mg} \mathrm{g}^{-1}$ para 
$101,3 \mathrm{mg} \mathrm{g}^{-1}$ com o tratamento, indicando melhora na capacidade da quitina em remover cobalto de soluções aquosas. Comparada a outros adsorventes já estudados para a adsorção de cobalto, como casca de avelã $-\mathrm{q}_{\mathrm{m}}=13,88$ (Demirbas, 2003) e fibra de polietileno tereftalato $\mathrm{q}_{\mathrm{m}}=27,2$ (Coskun et al., 2006), pode-se dizer que a quitina possui uma alta capacidade de adsorção, tornando-se ainda maior com o tratamento.

\section{CONCLUSÃO}

Com base nos resultados obtidos neste estudo, pode-se concluir que o tratamento utilizando $\mathrm{CO}_{2}$ supercrítico é uma alternativa interessante para melhorar as características da quitina como adsorvente, especialmente na sua capacidade de remover cobalto em soluções aquosas.

Através da análise dos parâmetros obtidos nos ajustes, pode-se dizer que o comportamento cinético foi mais bem descrito pelo modelo de pseudo-segunda ordem. $\mathrm{O}$ modelo de Langmuir representou melhor o equilíbrio de adsorção, demonstrando um aumento superior a $100 \%$ na capacidade de adsorção do material modificado em relação ao mesmo sem tratamento.

\section{REFERÊNCIAS}

COSKUN, R., et al. Adsorption of copper (II), nickel(II) and cobalt(II) ions from aqueous solution by methacrylic acid/acrylamide monomer mixture grafted poly(ethylene terephthalate) fiber. Sep. Purif. Technol. v., 49, p. 107-114, 2006.

DEMIBRAS, A. Heavy metal adsorption onto agro-based waste materials: A review. J.of Hazard. Mat., v. 157, p. 220-229, 2008.

FIGUEIREDO, S.A. et al. Natural waste materials containing chitin as adsorbents fortextile dyestuffs: Batch and continuous studies. Water Research, v. 39, p. 4143, 2005.

FRANCO, L. de O. et al. Heavy Metal Biosorption by Chitin and Chitosan Isolated From Cunninghamella Elegans (IFM 46109).Brazilian Journal of Microbiology, v. 35, p. 243, 2004.

JAVED, M. A. et al. Kinetic and Equilibrium Modeling of Pb(II) and Co(II) Sorption onto Rose Waste Biomass. Separation Science and Technology, v. 42, p. 3642, 2007.

LONGHINOTTI, E. et al. Adsorção de azul de metileno e croconato amarelo pelo biopolímeroquitina.Química Nova, v. 19, p. 221, 1996.

PRASHANTH, K.V. H.,THARANATHAN, R.N. Chitin/chitosan: modifications and their unlimited application potential - an overview. Trends in Food Science \& Technology, v. 18, p. 117, 2007.

SANTOS, J. C. dos. Extração com fluido supercrítico e suas aplicações na obtenção de produtos naturais.2011. 40 f. Trabalho de conclusão de curso(Graduação em Farmácia)Universidade Federal do Rio Grande do Sul, Porto Alegre, 2011. 
XI Congresso Brasileiro de Engenharia

Química em Iniciação Científica

Unicamp - Campinas - SP 19 a 22 de julho de 2015

SPINELli, A. V. et al. Cinética e Equilíbrio de Adsorção dos Oxiânions Cr (VI), Mo (VI) e Se (VI) pelo Sal de Amônio Quaternário de Quitosana. Polímeros: Ciência e Tecnologia, vol. 15, n 3, p. 218-223, 2005. 